\title{
Theory of electrochemistry at miniaturised interfaces between two immiscible electrolyte solutions
}

\author{
Damien W. M. Arrigan*,1 \& Grégoire Herzog ${ }^{2}$ \\ ${ }^{1}$ Nanochemistry Research Institute, Department of Chemistry, Curtin University, GPO Box U1987, \\ Perth, Western Australia, 6845, Australia. \\ ${ }^{2}$ Laboratoire de Chimie Physique et Microbiologie pour l'Environnement (LCPME), UMR 7564, CNRS \\ - Université de Lorraine, 405 rue de Vandoeuvre, 54600 Villers-les-Nancy, France. \\ * Email: d.arrigan@curtin.edu.au; telephone +61-8-9266-9735; fax: +61-8-9266-2300.
}

Summary. In this current opinion article on the electrochemistry of micro- and nano-interface between two immiscible electrolyte solutions, we present a summary of theoretical descriptions todate, rather than only of work in the past ca. 2 years. The opinion summarises the current state of art in the theory used to interpret and analyse this form of experimental electrochemistry, specifically addressing only simple ion transfer processes.

\section{The Interface between Two Immiscible Electrolyte Solutions}

Electrochemistry at the interface between two immiscible electrolyte solutions (ITIES) was first described by W. Nernst; subsequently the work of C. Gavach et al. and J. Koryta et al. popularised the topic and the acronym. Subsequently, the topic has developed into a distinct area of investigation (as summarised in some excellent reviews (e.g. Vanysek [1], Girault [2], Samec [3]). This topic is fundamentally important as it offers a means to measurement of the characteristics of ion-transfer reactions as well as providing a practical basis for the understanding and improvement of extraction processes, membrane technologies, chemical sensors and biomimetic systems.

\section{Miniaturisation}

The aim of miniaturisation of the ITIES, to microscale or to nanoscale, is to harness the benefits of such smaller interfaces. Primarily this is a diminished impact of uncompensated resistance on the experimental results, together with a decreased capacitance due to the smaller interface size. Additionally, the enhanced mass transport that derives from convergent diffusion enables steadystate measurements for kinetic studies. By use of glass pipettes, the ITIES was miniaturised to the micrometre scale $[4,5]$ and then the nanometre scale [6], with the prime motivation being to enable measurement of faster charge transfer processes at these interfaces.

Such miniaturised interfaces are prepared in two ways: by use of pipettes, usually in glass, or porous membranes as interface supports. Glass pipettes can be pulled [7], with laser heating systems, to provide openings of micrometre and nanometre dimensions. Location of the ITIES at the mouth of these pipettes is possible, with careful use of surface silanisation chemistry to control the glass surface properties to enable filling of the pipette interior with either organic phase or aqueous phase. Such pipette-based ITIES are usually employed to form single or dual ITIES. On the other hand, for some measurements, such as in sensors or bioanalytical systems, larger currents than are possible at microor nano-ITIES are needed. Then arrays [8] can be used which are prepared with inert membranes with through-holes in which the two electrolyte solutions meet to form the micro- or nano-ITIES. Materials and hole preparation methods for arrays of miniaturised ITIES include microporous polymer [8-10] 
and glass membranes [11] subjected to laser ablation, microporous silicon membranes $[12,13]$ formed by photolithography and reactive ion etching, and nanoporous silicon nitride using made by electronbeam lithography with reactive ion etching [14] or focused ion beam milling [15].

\section{Factors that influence electrochemistry at micro- or nano-ITIES}

The capacitance of the liquid-liquid interfaces ITIES scales with interfacial area so that miniaturised ITIES have lower RC time constants, thus enabling faster measurements. Nevertheless, the capacitance of such systems remains significant, not least because of the presence of two electrical double layers, one in each phase. Theoretical and experimental studies of the interface structure have converged to the idea that the dynamic interface consists of capillary waves, which averaged over time is similar to a mixed solvent region with thickness of ca. $1 \mathrm{~nm}[3,16]$. The use of membranes to pattern arrays of ITIES introduces additional capacitance, especially for membranes of nanometre thicknesses [17], which then places a limitation on the application of pulsed potential voltammetric methods.

Diffusion-controlled processes at micro- and nano-ITIES enable the application of microelectrode models for understanding of current processes. At single micro- or nano-ITIES located at the mouth of glass pipettes, the issue of width of the insulating region surrounding the ITIES is important, as the diffusion field will vary with thickness of the glass wall forming the pipette. The angle of the pipette wall will also influence the diffusion characteristics. At micro-ITIES arrays, the separation distance between elements of the array must be taken into consideration, in order to avoid, by design, diffusion zone overlap which will otherwise diminish the average current at each microlTIES in the array. This is usually achieved by use of the design rules established for microelectrode arrays $[18,19]$,

$$
\left.\delta \approx \sqrt{2 D \frac{\Delta\left(\Delta_{o}^{w} \varphi\right)}{v}} \quad \text { (eq. } 1\right)
$$

in which $\delta$ is the diffusion zone thickness, $D$ is the diffusion coefficient, $\Delta\left(\Delta_{o}^{w} \varphi\right)$ is the difference in potential differences between commencement of ion transfer and attainment of the steadystate, and $v$ is the voltammetric sweep rate. At nanoscale, however, it has been found that these design rules do not apply and individual nanoITIES $[14,20]$ in an array must be further separated from each other in order to be subjected to independent radial diffusion and achieve the maximum amperometric response [21-23].

The ability to measure rapid kinetics at the ITIES is a direct consequence of the increased mass transport rate (radial diffusion) and decreased capacitance. Typically, a Butler-Volmer-type model is used to relate the experimental current to the kinetic parameters [3],

$$
I_{i}=z F A\left(k_{f} c_{i}^{w}-k_{b} c_{i}^{o}\right) \quad(\text { eq. 2) }
$$

where $I_{i}$ is the current due to ion transfer, $z$ is the ion charge, $F$ the Faraday constant, $A$ the interfacial area, $c_{i}^{w}$ and $c_{i}^{o}$ are the ion concentrations in the water and organic phases, respectively, and $k_{f}$ and $k_{b}$ are the forward and backward rate constants, which are potential-dependent. As discussed by Samec [3], the determined rate constants for simple ion transfers have increased over the decades, perhaps as an indication of improved measurement methods. New theories of ion transfer kinetics at the ITIES were introduced to accommodate the capillary waves $[24,25]$ present at the interface and propose the formation of a water finger consistent with a hidden barrier for ion transfer [26, 27]. Marecek [28] used the common ion method [29] to determine ion transfer rate constants, achieving 
rate constants of ca. $0.5 \mathrm{~cm} \mathrm{~s}^{-1}$ for tetraethylammonium transfer between water and 1,2dichloroethane. Slower rate constants than expected $\left(100 \mathrm{~cm} \mathrm{~s}^{-1}\right)$ could be attributed to a slow relaxation of the interface, or even to the hidden barrier that was identified [26], attributed to formation of water fingers.

\section{Theory used to analyse experimental electrochemistry at micro- and nano-ITIES}

For a diffusion-controlled simple ion transfer process at a single pipette-based micro- or nano-ITIES, the steady-state, diffusion-controlled current $\left(I_{d}\right)$ developed in a voltammetric or amperometric experiment is described by the expression [30]

$$
I_{d}=4 x z F D c r
$$

which assumes a disc shaped interface is formed. In this equation, $z, D$ and $c$ are the charge, diffusion coefficient and bulk concentration of the transferring ion, respectively, $F$ is the Faraday constant, $r$ is the interface radius, and $x$ is the ratio of the inner and outer pipette tip radii, which accounts for the thickness of the pipette wall at the orifice. Use of this diffusion-controlled current model for characterisation of micro- and nano-ITIES is well established. For instance, recent experiments on neurotransmitter detection by ion transfer reactions at single nanoITIES employed this model in characterisation the nano-ITIES [31, 32]. Figure 1 shows voltammograms for the facilitated transfer of dopamine across the ITIES formed between water and 1,2-dichloroethane phase at the mouth of a pipette of radius $223 \mathrm{~nm}$. Using experimental current - concentration calibration graphs together with equation 3, the diffusion coefficient of dopamine was determined to be ca. $5 \times 10^{-10} \mathrm{~m}^{2} \mathrm{~s}^{-1}$ [32], in agreement with literature values. In situations where the wall of the glass pipette is tapered, the wall angle must be taken into consideration [29, 33-35] , leading to expressions for the time-dependent diffusion-controlled current variation with the wall angle, and hence the time to reach a steady-state.

At ITIES arrays formed in perforated membranes, diffusion controlled ion transfer currents at microor nano-disc ITIES are described by the expression

$$
I_{d}=N_{p} 4 z F D c r
$$

where $N_{p}$ is the number of pores in the membrane forming the ITIES, and the other parameters are as previously defined. Of course this is the well-known Saito equation [36] for diffusion-controlled current at a disc microelectrode, modified for the number of pores forming the interfaces of the array, and in this case assumes that all ITIES within the array are diffusionally-independent, as governed by eq. 1 for separation distances, and located at the mouths of the membrane pores. Typical examples of the application of this model to micro-ITIES array characterisation include those of laser-ablated polymer membrane-supported ITIES employed for the detection of heavy metals such as $\mathrm{Cd}^{2+}$ ions [37] and silicon membrane-supported micro-ITIES arrays for the detection of drug species [38]. These results are typical of those in the literature in which well-established diffusion-controlled current models are used to establish the response characteristics of experimental systems.

A major difference between microelectrodes (and their arrays) and micro-ITIES (and their arrays) is the asymmetry of diffusion processes occurring on forward and reverse cycles of a cyclic voltammogram: these are more different than the value of the diffusion coefficient of the species undergoing study. The geometry of the pores used to form the micro-ITIES has an important role. Depending on the pore length, pore wall angle, location of the interface relative to the pore mouth 
and, indeed, which phase fills the pores, different diffusion characteristics are obtained on the reverse sweep of a cyclic voltammogram. Taking into consideration these factors enables an analysis of the electrochemical response using published models [11]. Such diffusion zone differences are summarised in Figure 2 [39]. In the cases where the ITIES is located at the mouth of a micropore or micropipette, radial diffusion will occur for the transfer process from outside to inside the pore/pipette (ingress current), irrespective of which phase fills the pore/pipette. Then, due to the constraints imposed by the pore/pipette walls, linear diffusion will dominate on the reverse scan (egress current), leading to a different voltammetric shape for the forward and reverse ion transfer processes. For processes controlled by linear diffusion within the pipette/pore, the Randles-Sevcik equation applies,

$$
I_{p}=2.69 \times 10^{5} z^{3 / 2} A D^{1 / 2} c v^{1 / 2} \quad \text { (eq. 5) }
$$

(all parameters as previously defined) and it can be used to analyse the voltammetric response. The impact of pore wall angle (or pipette wall angle) can also be seen in the voltammetry and must be accounted for to achieve reliable kinetic data, for example. Specific expressions for the dependence of the egress current on the wall angle are available [29, 33, 34, 40, 41], such as eq. 6 [41],

$$
I_{d} \approx \pi z F D \operatorname{crsin} \theta \quad \text { (eq. 6) }
$$

in which $\Theta$ is the wall angle, and the other parameters are as previously defined.

\section{Finite element simulations in understanding such electrochemistry}

Many groups use finite element simulations to guide the development of micro- and nano-ITIES systems [39], in which simulation models are used to extract information about the response characteristics, in addition to that achieved by comparison to simple models derived from microelectrode systems. For instance, simple ion transfer at the nano-ITIES was used to develop the experimental method of common ion voltammetry for kinetic determinations [29]. Modelling of mass transport behaviour at nanolTIES within an array was used to evaluate the role of diffusion zone overlap in attenuating the amperometric responses to ion transfer processes [42]. Similarly, mass transport modelling was used to examine hole geometry effects in arrays of microholes formed in glass membranes by laser ablation [11]. In this case, asymmetric microholes were formed by the laser ablation process and location of the ITIES at either the wider or the narrower opening of the hole elicited a characteristic difference in the voltammetry which was explained by the mass transport effect. Such modelling processes invariably can employ a reversible ion transfer process, as well as serve as a probe of kinetic effects on the voltammetry [29]. A further important consideration investigated by simulation models is the influence of the pipette wall surface charge, which have shown that the ion-transfer current is higher at the edge of the ITIES close to the charged glass wall [29].

\section{Analytical models at micro- or nano-ITIES}

The description of ion transfer at micro- and nano-ITIES using analytical models that account for the asymmetric diffusion profiles referred to above has been of interest as it can provide a quicker way to analyse experimental behaviour relative to theoretical expectations. Displayed in Figure $\mathbf{3}$ are the diffusion profiles outside a channel mouth and within a channel that may be of micro- or nanometre dimensions. The ITIES located at the channel mouth in this case will be subjected to the described 
diffusion profiles depending on ion movement into the channel our out of the channel. This effect has been modelled analytically by Molina et al. [43-45] to determine the voltammetric features of such ITIES at micro- or nanoscales. In that series of papers, application of such analytical models using pulse techniques like chronoamperometry, staircase cyclic voltammetry, square wave voltammetry, staircase linear sweep voltammetry, normal pulse voltammetry and double differential pulse voltammetry were investigated. The analytical models account for the asymmetric diffusion processes at a capillary tip, employ reversible charge transfer conditions, with very good agreement with simulation results. The models reveal the dependence on ratio of the ion diffusion coefficients in the two phases, the size of the capillary forming the ITIES, and the timescale of the experiment [43] importantly, for a reversible ion transfer at a micro- or nano-ITIES, the transfer potentials shift with the timescale (scan rate, frequency), unlike the case at millimetre-scale or larger ITIES. Another interesting observation from this theoretical model is the variation of the concentration profile across the micro- or nano-channel width [44], which cannot be determined by application of equations 3-5.

\section{Conclusions}

Theoretical aspects of ion-transfer electrochemistry at miniaturised ITIES (micro- or nanoscales) borrow heavily from the electrochemistry at microelectrodes and microelectrode arrays. As a result, mass-transport-controlled electrochemistry at micro- and nano-ITIES is well established and employed for characterisation and detection purposes. New analytical models are emerging that help in further understanding of observed behaviour as well as disclosing new features of the electrochemistry. However, truly comprehensive models that incorporate mass transport, kinetics and capacitance, to enable a complete characterisation of a system under dynamic electrochemical conditions have yet to appear and, ideally, modellers with access to the necessary computing facilities will bring such gifts to experimentalists in the coming years.

\section{Acknowledgements}

DWMA thanks the Australian Research Council (DP130102040) for financial support. GH is grateful to Campus France for funding his visit to Curtin University (PHC FASIC 2016, 37186PC)

\section{References}

[1] P. Vanysek, L.B. Ramirez, Interface between two immiscible liquid electrolytes: A review, J. Chil. Chem. Soc., 53 (2008) 1455-1463.

[2] H.H. Girault, Electrochemistry at Liquid-Liquid Interfaces, in: A.J. Bard, C.G. Zoski (Eds.) Electroanalytical Chemistry, A Series of Advances, Vol 23, Dekker, New York, 2010, pp. 1-104.

[3] Z. Samec, Dynamic electrochemistry at the interface between two immiscible electrolytes, Electrochim. Acta, 84 (2012) 21-28.

[4] G. Taylor, H.H.J. Girault, Ion transfer reactions across a liquid-liquid interface supported on a micropipette tip, J. Electroanal. Chem., 208 (1986) 179-183.

[5] J.A. Campbell, H.H. Girault, Steady-state current for ion transfer reactions at a micro liquid liquid interface, J. Electroanal. Chem., 266 (1989) 465-469.

[6] Y.H. Shao, M.V. Mirkin, Fast kinetic measurements with nanometer-sized pipets. Transfer of potassium ion from water into dichloroethane facilitated by dibenzo-18-crown-6, J. Amer. Chem. Soc., 119 (1997) 8103-8104. 
[7] S.J. Liu, Q. Li, Y.H. Shao, Electrochemistry at micro- and nanoscopic liquid/liquid interfaces, Chem. Soc. Rev., 40 (2011) 2236-2253.

[8] H.J. Lee, P.D. Beattie, B.J. Seddon, M.D. Osborne, H.H. Girault, Amperometric ion sensors based on laser-patterned composite polymer membranes, J. Electroanal. Chem., 440 (1997) 73-82.

[9] H.J. Lee, C. Beriet, H.H. Girault, Stripping voltammetric determination of choline based on microfabricated composite membrane, Anal. Sci., 14 (1998) 71-77.

[10] H.J. Lee, H.H. Girault, Amperometric ion detector for ion chromatography, Anal. Chem., 70 (1998) 4280-4285.

[11] E. Alvarez de Eulate, J. Strutwolf, Y. Liu, K. O’Donnell, D.W.M. Arrigan, An Electrochemical Sensing Platform Based on Liquid-Liquid Microinterface Arrays Formed in Laser-Ablated Glass Membranes, Anal. Chem., 88 (2016) 2596-2604.

* used a finite element simulation model to explain the role of pore wall slope on the voltammetric behaviour.

[12] R. Zazpe, C. Hibert, J. O'Brien, Y.H. Lanyon, D.W.M. Arrigan, Ion-transfer voltammetry at silicon membrane-based arrays of micro-liquid-liquid interfaces, Lab Chip, 7 (2007) 1732-1737.

[13] J. Strutwolf, M.D. Scanlon, D.W.M. Arrigan, Electrochemical ion transfer across liquid/liquid interfaces confined within solid-state micropore arrays - simulations and experiments, Analyst, 134 (2009) 148-158.

[14] M.D. Scanlon, J. Strutwolf, A. Blake, D. Iacopino, A.J. Quinn, D.W.M. Arrigan, Ion-Transfer Electrochemistry at Arrays of Nanointerfaces between Immiscible Electrolyte Solutions Confined within Silicon Nitride Nanopore Membranes, Anal. Chem., 82 (2010) 6115-6123.

[15] M. Sairi, N. Chen-Tan, G. Neusser, C. Kranz, D.W.M. Arrigan, Electrochemical Characterisation of Nanoscale Liquid jLiquid Interfaces Located at Focused Ion Beam Milled Silicon Nitride Membranes, ChemElectroChem, 2 (2015) 98-105.

[16] P. Peljo, H.H. Girault, Liquid/Liquid Interfaces, Electrochemistry at, Encyclopedia of Analytical Chemistry, John Wiley \& Sons Ltd., 2006.

[17] M. Sairi, J. Strutwolf, R.A. Mitchell, D.S. Silvester, D.W.M. Arrigan, Chronoamperometric response at nanoscale liquid-liquid interface arrays, Electrochim. Acta, 101 (2013) 177-185.

[18] T.J. Davies, S. Ward-Jones, C.E. Banks, J. del Campo, R. Mas, F.X. Munoz, R.G. Compton, The cyclic and linear sweep voltammetry of regular arrays of microdisc electrodes: Fitting of experimental data, J. Electroanal. Chem., 585 (2005) 51-62.

[19] T.J. Davies, R.G. Compton, The cyclic and linear sweep voltammetry of regular and random arrays of microdisc electrodes: Theory, J. Electroanal. Chem., 585 (2005) 63-82.

[20] M. Rimboud, R.D. Hart, T. Becker, D.W.M. Arrigan, Electrochemical behaviour and voltammetric sensitivity at arrays of nanoscale interfaces between immiscible liquids, Analyst, 136 (2011) 46744681.

[21] N. Godino, X. Borrise, F.X. Munoz, F.J. del Campo, R.G. Compton, Mass Transport to Nanoelectrode Arrays and Limitations of the Diffusion Domain Approach: Theory and Experiment, J. Phys. Chem. C, 113 (2009) 11119-11125.

* used a finite element simulation model to examine the diffusion zone interaction at nanoelectrode arrays, and is directly relevant to voltammetry at nano-ITIES arrays.

[22] Y. Liu, M. Sairi, G. Neusser, C. Kranz, D.W.M. Arrigan, Achievement of Diffusional Independence at Nanoscale Liquid Liquid Interfaces within Arrays, Anal. Chem., 87 (2015) 5486-5490.

[23] Y. Liu, A. Holzinger, P. Knittel, L. Poltorak, A. Gamero-Quijano, W.D.A. Rickard, A. Walcarius, G. Herzog, C. Kranz, D.W.M. Arrigan, Visualization of Diffusion within Nanoarrays, Anal. Chem., 88 (2016) 6689-6695.

[24] I. Benjamin, Mechanism and dynamics of ion transfer across a liquid-liquid interface, Science, 261 (1993) 1558-1560.

[25] J.J. Karnes, I. Benjamin, Geometric and energetic considerations of surface fluctuations during ion transfer across the water-immiscible organic liquid interface, J. Chem. Phys., 145 (2016) 014701. 
* used molecular dynamics modelling to study chloride transfer across at the waternitrobenzene interface in the presence of surface fluctuations.

[26] N. Kikkawa, L. Wang, A. Morita, Microscopic Barrier Mechanism of Ion Transport through LiquidLiquid Interface, J. Amer. Chem. Soc., 137 (2015) 8022-8025.

$* *$ used molecular dynamics simulations to examine ion transfer across the oil-water interface, identifying the formation and disruption of water fingers into the oil phase accompanying ion transfer.

[27] N. Kikkawa, L. Wang, A. Morita, Computational study of effect of water finger on ion transport through water-oil interface, J. Chem. Phys., 145 (2016).

[28] A. Trojanek, V. Marecek, Z. Samec, Temperature effect in the ion transfer kinetics at the microinterface between two immiscible electrolyte solutions, Electrochim. Acta, 180 (2015) 366-372. [29] P.J. Rodgers, S. Amemiya, Y.X. Wang, M.V. Mirkin, Nanopipet Voltammetry of Common lons across the Liquid-Liquid Interface. Theory and Limitations in Kinetic Analysis of Nanoelectrode Voltammograms, Anal. Chem., 82 (2010) 84-90.

* used simulations to study wall angle effects and wall surface charge effects at nanolTIES.

[30] S. Amemiya, Y. Wang, M.V. Mirkin, Nanoelectrochemistry at the liquid/liquid interfaces, in: R.G. Compton \& J.D. Wadhawan (Eds.), Specialist Periodical Reports - Electrochemistry, Royal Society of Chemistry Cambridge, 2014, pp. 1-43.

[31] M.L. Colombo, J.V. Sweedler, M. Shen, Nanopipet-Based Liquid-Liquid Interface Probes for the Electrochemical Detection of Acetylcholine, Tryptamine, and Serotonin via Ionic Transfer, Anal. Chem., 87 (2015) 5095-5100.

[32] M.L. Colombo, S. McNeil, N. Iwai, A. Chang, M. Shen, Electrochemical Detection of Dopamine via Assisted Ion Transfer at Nanopipet Electrode Using Cyclic Voltammetry, J. Electrochem. Soc., 163 (2016) H3072-H3076.

* used ion transfer voltammetry with applications of mass transport-controlled current models to characterise neurotransmitter transfer across the nano-ITIES.

[33] N. Nishi, S. Imakura, T. Kakiuchi, A digital simulation study of steady-state voltammograms for the ion transfer across the liquid-liquid interface formed at the orifice of a micropipette, J.

Electroanal. Chem., 621 (2008) 297-303.

[34] P.J. Rodgers, S. Amemiya, Cyclic voltammetry at micropipet electrodes for the study of iontransfer kinetics at liquid/liquid interfaces, Anal. Chem., 79 (2007) 9276-9285.

[35] Y. Wang, T. Kakiuchi, Y. Yasui, M.V. Mirkin, Kinetics of Ion Transfer at the Ionic Liquid/Water Nanointerface, J. Amer. Chem. Soc., 132 (2010) 16945-16952.

[36] Y. Saito, A theoretical study on the diffusion current at the stationary electrodes of circular and narrow band types, Rev. Polarog. (Japan), 15 (1968) 177-187.

[37] A. Mastouri, S. Peulon, D. Farcage, N. Bellakhal, A. Chausse, Perfect additivity of microinterface arrays for liquid-liquid measurements: Application to cadmium ions quantification, Electrochim. Acta, 120 (2014) 212-218.

[38] M. Sairi, D.W.M. Arrigan, Electrochemical detection of ractopamine at arrays of micro-liquid vertical bar liquid interfaces, Talanta, 132 (2015) 205-214.

[39] J. Josserand, J. Morandini, H.J. Lee, R. Ferrigno, H.H. Girault, Finite element simulation of ion transfer reactions at a single micro-liquid I liquid interface supported on a thin polymer film, J.

Electroanal. Chem., 468 (1999) 42-52.

* introduced finite element simulations of electrochemistry at single micro-ITIES, taking into account interface location within microholes in a polymer membrane.

[40] Y.X. Wang, J. Velmurugan, M.V. Mirkin, P.J. Rodgers, J. Kim, S. Amemiya, Kinetic Study of Rapid Transfer of Tetraethylammonium at the 1,2-Dichloroethane/Water Interface by Nanopipet Voltammetry of Common lons, Anal. Chem., 82 (2010) 77-83.

[41] Q. Li, S.B. Xie, Z.W. Liang, X. Meng, S.J. Liu, H.H. Girault, Y.H. Shao, Fast Ion-Transfer Processes at Nanoscopic Liquid/Liquid Interfaces, Angew. Chem.-Int. Edit., 48 (2009) 8010-8013. 
[42] Y. Liu, J. Strutwolf, D.W.M. Arrigan, Ion-Transfer Voltammetric Behavior of Propranolol at Nanoscale Liquid-Liquid Interface Arrays, Analy. Chem., 87 (2015) 4487-4494.

[43] A. Molina, E. Laborda, R.G. Compton, Cyclic and Square-Wave Voltammetry at Diffusionally Asymmetric Microscopic and Nanoscopic Liquid-Liquid Interfaces: A Simple Theoretical Approach, J.

Phys. Chem. C, 118 (2014) 18249-18256.

** developed analytical model incorporating reversible ion transfer and asymmetric diffusion profiles to examine ion transfer processes at ITIES located at the mouths of microor nano-channels.

[44] A. Molina, E. Laborda, R.G. Compton, An approximate theoretical treatment of ion transfer processes at asymmetric microscopic and nanoscopic liquid-liquid interfaces: Single and double potential pulse techniques, Chem. Phys. Lett., 597 (2014) 126-133.

[45] A. Molina, E. Laborda, J. Gonzalez, R.G. Compton, Linear Sweep and Cyclic Voltammetries of Reversible Ion Transfer Processes at Macro- and Microcapillaries under Transient Regime, Electroanalysis, 27 (2015) 93-100. 


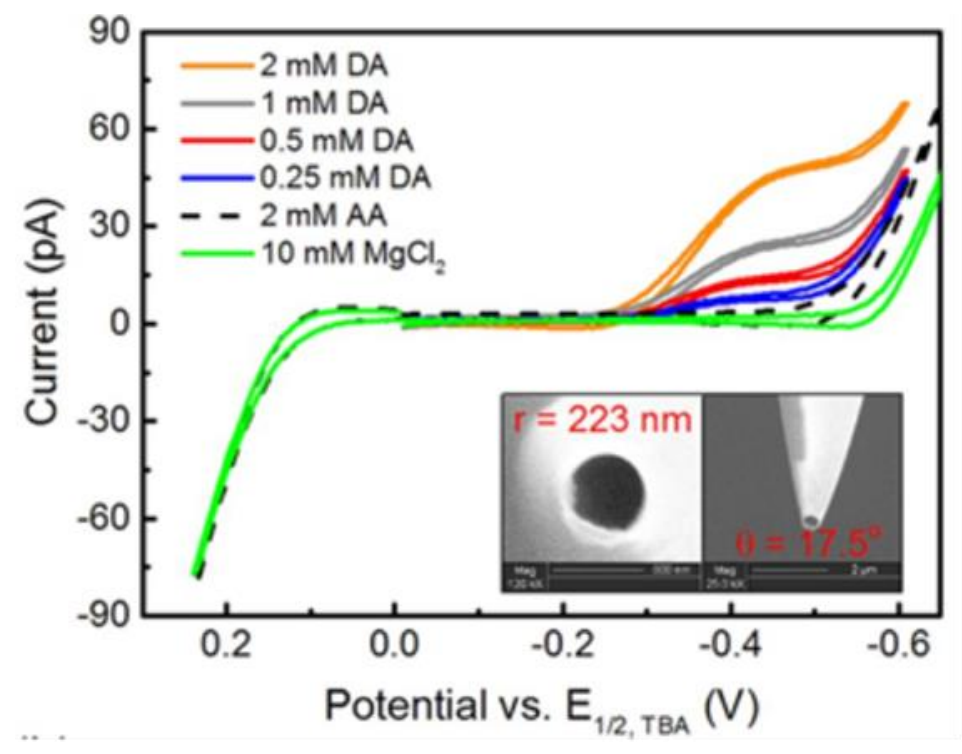

Figure 1. Cyclic voltammograms at an ITIES formed at the mouth of a $223 \mathrm{~nm}$ radius glass pipette, in which the aqueous phase contains ascorbic acid (AA, $2 \mathrm{mM}$ ) with subsequent additions of dopamine (DA, 0.25-2 mM). Dibenzo-18-crown-6 is present in the organic phase to facilitate the transfer of DA. Reproduced from J. Electrochem. Soc., 163 (2016) H3072-H3076, published open access under creative commons license https://creativecommons.org/licenses/by-nc-nd/4.0/. 

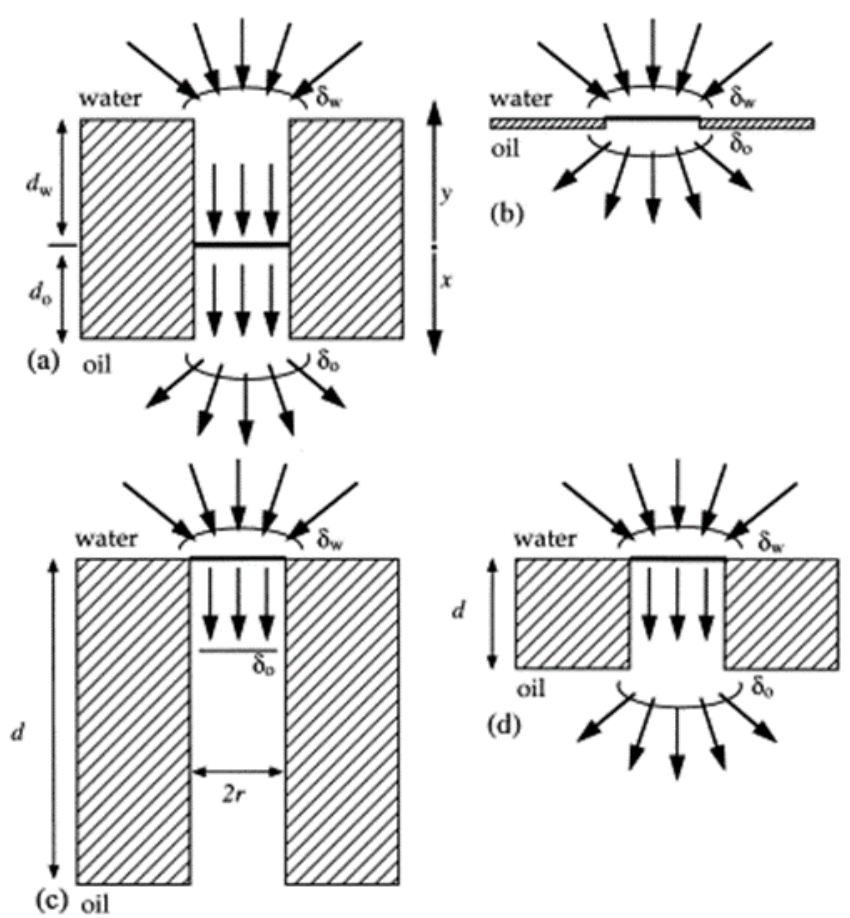

Figure 2. Illustration of diffusion domains (as indicated by the directional arrows) for micro-ITIES formed at holes within membranes. (a) Recessed disc micro-interface, (b) Quasi inlaid disc micro-interface, (c) inlaid disc micro-interface formed at a long micro-hole, (d) inlaid disc micro-interface formed at a long short-hole. Reproduced with permission from J. Electroanal. Chem., 468 (1999) 42-52. Copyright (1999) Elsevier. 


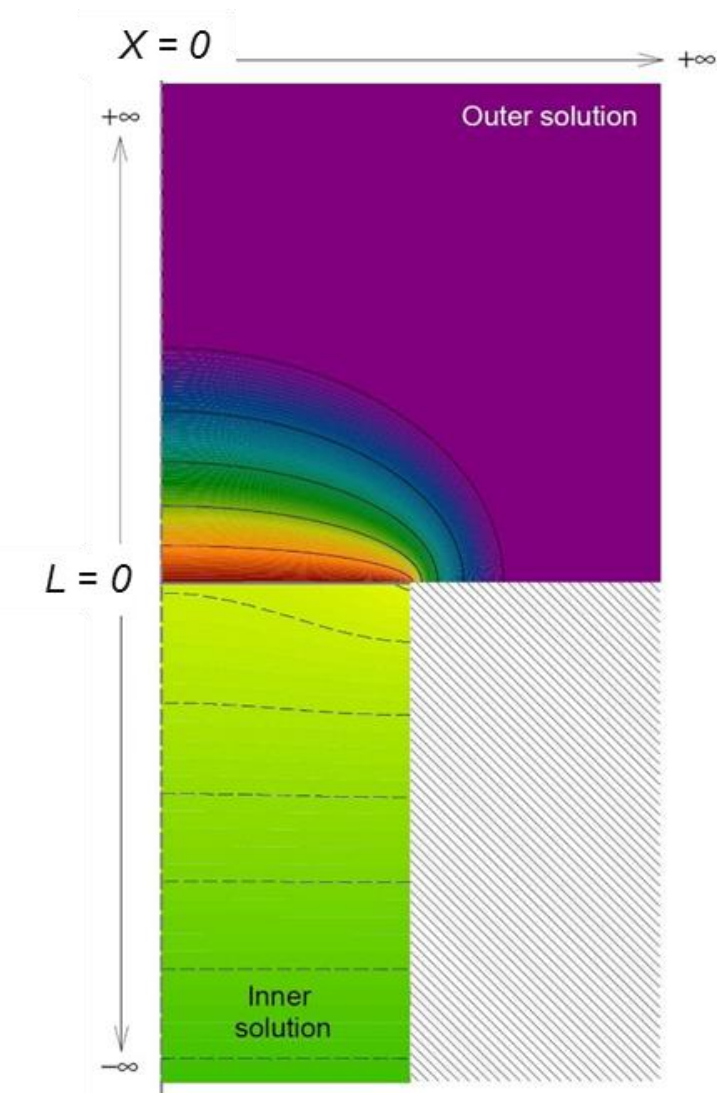

Figure 3. Illustration of the diffusion fields that form following ion transfer across a liquidliquid interface located at a micro- or nano-channel mouth used in the analytical modelling of ion-transfer voltammetry. $L=0$ is the interface location. Adapted with permission from $J$. Phys. Chem. C, 118 (2014) 18249-18256. Copyright (2014) American Chemical Society. 